\title{
Code System
}

National Cancer Institute

\section{Source}

National Cancer Institute. Code System. NCI Thesaurus. Code C70895.

A systematized collection of concepts that define corresponding codes. 